\title{
Isolated Mesenteric Tuberculosis
}

\section{Roshan Ghimire, Uttam Laudari and Deepak Raj Singh}

Department of Surgery, Kathmandu Medical College Teaching Hospital, Kathmandu, Nepal.

\section{ABSTRACT}

Introduction: Abdominal tuberculosis is common in developing world, and abdominal tuberculosis is most common presentation of extra pulmonary tuberculosis. High index of suspicion is needed in patient from endemic area with chronic abdominal pain and constitutional symptom for diagnosis of abdominal tuberculosis. In abdominal tuberculosis although intestinal, cecal and lymph node involvement are common, isolated mesenteric tuberculosis is rare presentation and very few cases has been reported. Here we are presenting rare case of a young male with isolated mesenteric tuberculosis who presented with abdominal mass.

Keywords: abdominal mass; mesentery; tuberculosis

\section{INTRODUCTION}

Abdominal tuberculosis is very common in developing world, and it is most common form of extra abdominal tuberculosis ${ }^{1} .8583$ per 100000 new cases of extra pulmonary tuberculosis were reported in Nepal in $2014^{2}$. Judicious use of steroids, immunosuppressant, chemotherapeutic drugs, and wide spread increasing prevalence of Mycobacterium avium-intracellularae due to Human immune deficiency viruses has increases the incidence of abdominal tuberculosis ${ }^{3}$.

The terminal ileum and cecum is the most commonly involved site. The most common presentation is abdominal pain, diarrhoea, obstruction, hematochezia and palpable mass in abdomen or can be incidental finding during laparoscopic/ laparotomy procedure ${ }^{4}, 5$. Abdominal tuberculosis can be a significant cause of morbidity and mortality, due to its unusual presentation, and high index of suspicion is needed in endemic zone ${ }^{6}$. The incidence of peritoneal tuberculosis is $3-4 \%$ of all form of tuberculosis, and isolated mesenteric tuberculosis, presenting as abdominal lump is a rare entity few cases has been reported till date ${ }^{7,8}$. Here we present a case of 24 year old male who was refractory to medical therapy initially and had diagnostic dilemma on imaging and laparoscopy. He underwent surgical intervention and was diagnosed as isolated mesenteric tuberculosis and doing well under anti tubercular therapy.

\section{CASE REPORT}

Twenty seven years old male presented with a history of pain and mass in the right side of the abdomen for three months and had severe episodic pain, insidious, progressive in nature, associated with loss of appetite and nausea. His past medical history, family history and drug history were not significant. His general condition was fair abdominal examination revealed an ill-defined mass $6 \mathrm{~cm} \times 5 \mathrm{~cm}$ in the

Correspondence: Roshan Ghimire, Department of Surgery, Kathmandu Medical College, Kathmandu, Nepal. Email: roshanghimire@mail.com 


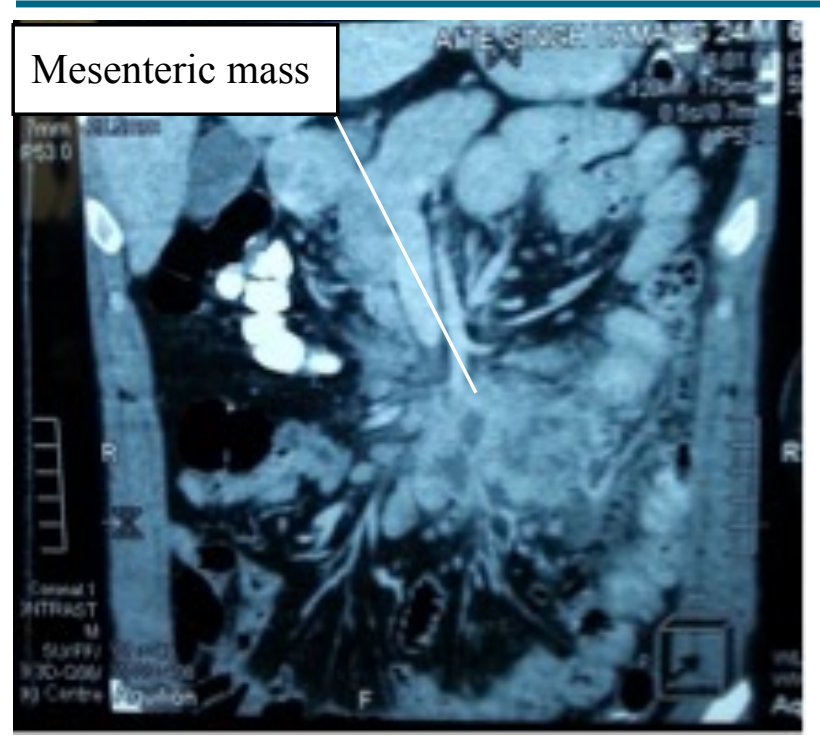

Figures 1: CECT abdomen

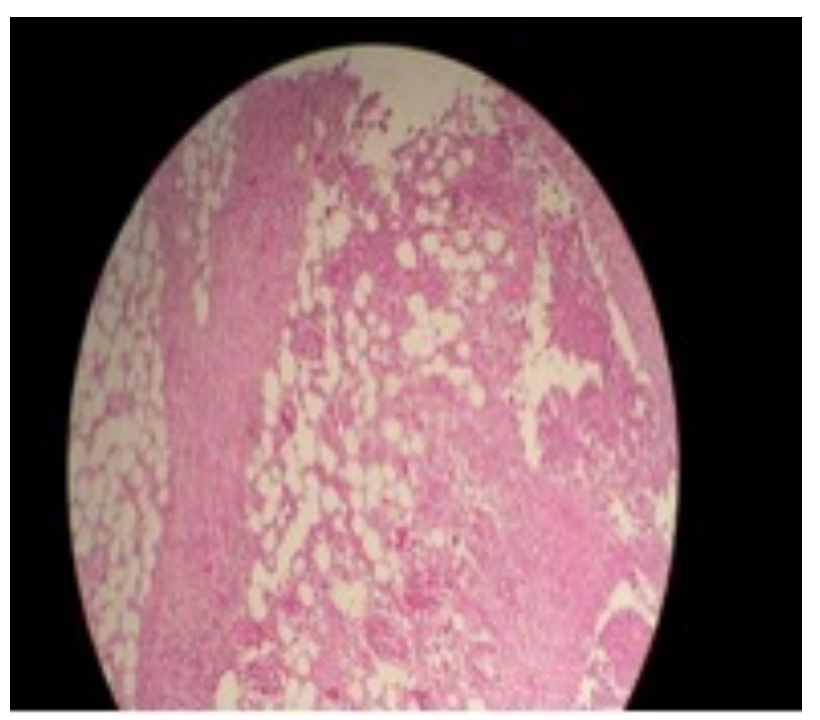

Figures 3 :microscopic features of the mass

left flank and left upper quadrant which was non tender, dull on percussion, less prominent on knee chest position, respiratory and cardiovascular examination were unremarkable.

His haematological and biochemical, chest $\mathrm{x}-$ ray, Mantoux test and sputum profile were all within in normal limit. ESR was $25 \mathrm{~mm} /$ hour. On imaging studies, ultrasound of abdomen and pelvis was within normal limit. CT scan revealed ill-defined enhancing soft tissue lesion in omentum and mesentery of the umbilical and left lumbar abdominal cavity

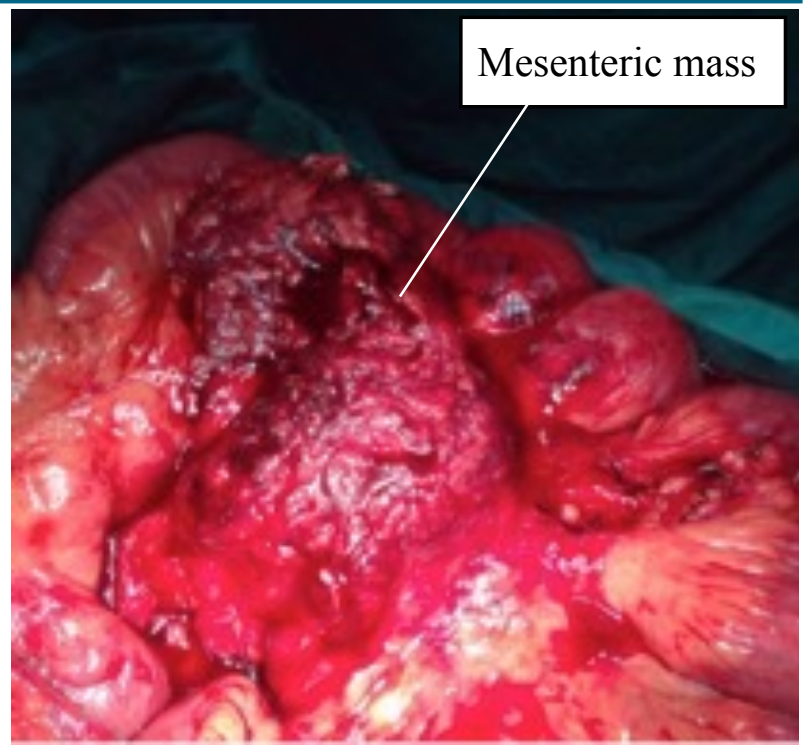

Figures 2:Gross features of the mass

with focal stranding of the omental and mesenteric fat with differential diagnosis of mesenteric/omental panniculitis of omental infarction. (Fig. 1)

Patient was refractory to medical therapy and with differential diagnosis of omental torsion and mesenteric cyst; we proceeded for diagnostic laparoscopy which revealed a mass around proximal jejunum covered by omentum which could not be mobilized. Exploratory laparotomy was performed which revealed a 10 $\mathrm{cm} \times 10 \mathrm{~cm} \times 3 \mathrm{~cm}$ solid mass on the mesentery in proximal jejunum with minimal ascitis and hyperaemic adjacent jejunum and nearby lymph nodes were normal.

On gross examination (Figure-2) mesentery showed an irregular mass confined within the mesentery measuring $12 \times 9 \times 6 \mathrm{~cm}$ with normal appearing intestinal mucosa, cut surface of mass was solid with brownish black discoloration, with unremarkable small intestine. Microscopic features were consistent with granulomatous inflammation with reactive changes in the lymph node and possible differential diagnosis of deep mycosis and tuberculosis was made. TB-PCR of the 
specimen was positive for Mycobacterium Tuberculosis. He was started on Category I Antitubercular drugs according to DOTSNepal. Patient is now symptom free and doing well in follow up.

\section{DISCUSSION}

We presented the case of young man with isolated mesenteric tuberculosis with nonspecific clinical features, abdominal lump, inconclusive CT scan and diagnostic laparoscopy; initially managed conservatively as panniculitis which usually resolves with conservative management ${ }^{9}$, later confirmed to be tuberculosis and responsive to therapy. The high index suspicion is needed endemic region for early diagnosis and treatment.

Peritoneal tuberculosis is not common presentation than other form of abdominal tuberculosis accounting for $3-4 \%$ of all form ${ }^{6,8}$. The presentation can be acute and chronic, with wet ascitic type being most common form of presentation than fixed fibrotic and dry plastic variety 6 . The triad of fever, ascitis and abdominal pain are present in $70 \%$ of patients with abdominal tuberculosis so diagnosis can be difficult in remaining patient with nonspecific clinical features and no radiological and laboratory findings ${ }^{10}$.

Nos P et al. in 1992 described similar cases of isolated mesenteric tuberculosis as first manifestation in HIV infected individuals, who presented with fever, pain abdomen and swelling. Patient had huge mesenteric abscess with thick capsule multiple septations and caseous material as in tubercular abscess; however he had no any abdominal lymphadenopathy as in our case ${ }^{11}$. Yutaka Tomizawa et al in 2013 reported similar case of 36 year old immunocompetent man as in our case with normal chest X-ray and negative MJSBH Vol 15 Issue 2 July-Dec 2016
PPD test as in our case. However presentation was different i.e. with moderate ascitis, prominent mesenteric and retroperitoneal lymph nodes, with omental infiltration causing small bowel obstruction ${ }^{12}$. In a study conducted by Cengiz Bolukbas et al among 88 HIV seronegative patients from 1994-2008, $\mathrm{n}=78(88.6 \%)$ had abnormality in ultrasound, whereas CT scan detected ascitis, lymphadenopathy and gut wall thickness and other abnormality in $81.8 \%$.Isolated involvement of peritoneum was $71 \%$ in patients with ascitis $(n=24)^{13}$. However in our case neither ultrasound nor CT scan could detect any abnormalities in favour of abdominal tuberculosis. We emphasize considering abdominal tuberculosis in any patient with abdominal lump despite of predisposing factors and presentation can be varied.

Routine blood investigation may show anemia, relative lymphocytosis, raised ESR, hypoalbuminemia ${ }^{4}$. Most of the patient will have positive tuberculin test, but the diagnostic value is very low as differentiation between active disease and previous sensitization/ vaccination cannot be made ${ }^{4,14}$. Abnormal chest radiograph is seen in $50 \%$ of cases $^{14}$. However in our case all the haematological, biochemical and immunological and chest xray were unremarkable. Ascitic fluid SAAG will be less than $1.1 \mathrm{~g} / \mathrm{dl}$, with presence of erythrocytes, increased leucocytes with predominant lymphocytes. Ascitic fluid adenosine deaminase is highly sensitive and specific for tuberculous peritonitis ${ }^{14}$. Acid fast stain and culture positive for organisms in ascitic fluid is positive in less than $3 \%$ and less than $20 \%$ of cases. The PCR from necrotizing granulomatous inflammation, including 
gastrointestinal tract and peritoneum can yield good results up to $85 \%$ than compared to 15.3 $\%$ and $24.4 \%$ by Ziehl-Neelsen stain and culture respectively ${ }^{15}$ which was positive in our case. Abdominal ultrasound and CT scan may aid in diagnosis but has less sensitivity and specificity. CT scan will demonstrate thickened and nodular mesentery with mesenteric lymphadenopathy, omental thickening with irregular soft tissue densities and ascitis with fine septations though such findings were not seen in our case $e^{4,10,14}$. Diagnostic laparoscopy with biopsy from peritoneum is gold standard in diagnosis peritoneal tuberculosis, sensitivity and specificity both being $100 \%{ }^{10}$. Findings consistent with peritoneal tuberculosis are thickened, hyperaemic peritoneum with ascites and whitish granular nodules approximately $5 \mathrm{~mm}$ scattered over the parietal and visceral peritoneum and markedly thickened parietal peritoneum with possibly yellowish nodules and cheesy material with thick multiple adhesions ${ }^{10,14}$. However we could not appreciate any abnormal findings during laparoscopy and so biopsy could not be taken.

\section{CONCLUSIONS}

Isolated mesenteric tuberculosis can be one of the unusual presentations of abdominal mass in endemic region like our which bears challenges to clinician and radiologist in diagnosis and treatment. High index of suspicion is required for timely diagnosis and treatment. Diagnostic laparoscopy and biopsy is the gold standard modality for diagnosis and patient have good outcome with medical therapy.

\section{REFERENCES}

1. National Tuberculosis Center. National Tuberculosis Programme, A Clinical Manual for Nepal. 2009;(Third edition). Available from: http://nepalntp.gov.np/theme/images/ uploads/13738740481inical_Manual.pdf

2. WHO. Tuberculosis country profile [Internet]. 2014. Available from: https:// extranet.who.int/sree/Reports? op $=$ Replet\&name=\%2FWHO_HQ_Reports $\% 2$ F G $2 \% 2$ F P R O D \% 2 F E X T $\% 2$ FTBCountryProfile \&ISO2 $=$ NP\&LAN $=$ EN\&outtype $=$ pdf

3. Norman S. Williams, P. Ronan O'Connell CJKB. Bailey and Love's Short Practice of Surgery. 2009.

4. Kapoor VK. Abdominal tuberculosis. Postgrad Med J [Internet]. 1998 Aug [cited 2016 Feb 19];74(874):459-67. Available from: http://www.pubmedcentral.nih.gov/ a r t i c 1 e r e $n$ d e r. f c g i ? artid $=2360888 \&$ tool $=$ pmcentrez\&rendertyp $\mathrm{e}=$ abstract

5. Kasper Hauser, Braunwald longo FJ. Harrison's Principles of Internal Medicine. 16th ed. Dennis L. Kasper, Anthony s. Fauci, Dan L. Longo, Eugene Braunwald, Stephen L. Hauser JLJ, editor. McGrawHill; 2005. 957-959 p.

6. Debi U, Ravisankar V, Prasad KK, Sinha SK, Sharma AK. Abdominal tuberculosis of the gastrointestinal tract: Revisited. World J Gastroenterol. 2014;20(40):14831-40. DOI: https://doi.org/10.3748/wjg.v20.i40.14831

7. Anand P, Sushmita C. Isolated Mesenteric Tuberculosis presented as Abdominal Mass. Correspondence : Natl J Med Res Case Rep. 2012;389-90.

8. Clancy C, Bokhari Y, Neary PM, Joyce M. Diagnosing peritoneal tuberculosis. BMJ Case Rep. 2013;2013:4-5. DOI: https://doi.org/10.1136/bcr-2013-009871

9. Tonerini M, Calcagni F, Lorenzi S, Scalise P, Grigolini A, Bemi P. Omental infarction and its mimics: imaging features of acute abdominal conditions presenting with fat stranding greater than the degree of bowel wall thickening. Emerg Radiol [Internet]. 2015 Aug [cited 2016 Mar 13];22(4):431-6. Available from: http:// www.ncbi.nlm.nih.gov/pubmed/25725796 
10. Abdelaal A, Alfkey R, Abdelaziem S, Abunada M, Alfaky A, Ibrahim WH, et al. Role of laparoscopic peritoneal biopsy in the diagnosis of peritoneal tuberculosis. A seven-year experience. Chirurgia (Bucur) [Internet]. Jan [cited 2016 Feb 20];109(3): 330-4. Available from: http:// www.ncbi.nlm.nih.gov/pubmed/24956337

11. Nos P, Ricart C, García E, Moles JR, Lacruz J, Berenguer J. [Isolated mesenteric tuberculosis as the first manifestation of AIDS]. Rev espa-ola enfermedades Dig organo Of la Soc Espa-ola Patol Dig [Internet]. 1992 Jul [cited 2016 Feb 20]; 82(1):59-60. Available from: http:// www.ncbi.nlm.nih.gov/pubmed/1520554

12. Tomizawa Y, Yecies EB, Craig FE, Sohnen A. Peritoneal tuberculosis in an immunocompetent, unknown risk patient. Case Rep Gastrointest Med. 2013;2013:680763. DOI: https://doi.org/10.1155/2013/680763

13. Bolukbas C, Bolukbas FF, Kendir T, Dalay $\mathrm{R}$ a, Akbayir N, Sokmen MH et al. Clinical presentation of abdominal tuberculosis in HIV seronegative adults. BMC Gastroenterol. 2005;5:21. DOI:

https://doi.org/10.1186/1471-230X-5-21

14. Townsend Courtney M, Beauchamp R. Daniel, Evers B, Mark MKL. Sabiston Text book of Surgery: The Biological Basis of Modern Surgical Pratice. 19th ed. Elsevier Inc.; 2012. 1101-1102 p.

15. Chuttani HK, Sarin SK. Intestinal tuberculosis. Indian J Tuberc. 1985;32(3): 117-25. 